\title{
Lack of adverse effects during a target animal safety trial of extended-release buprenorphine in Fischer 344 rats
}

\author{
Alan Cowan, $P h D^{1}$, Rachel Sarabia-Estrada, DVM, $P h D^{2}$, Gina Wilkerson, DVM ${ }^{3}$, \\ Patrick McKnight, $P h D^{4}$ \& Michael Guarnieri, $P h D^{2}$
}

Extended-release buprenorphine is an effective analgesic in laboratory animals, and its safety has been established in mice but not in rats. The authors used a target animal safety trial to evaluate the safety of extended-release buprenorphine in rats. Fischer 344 rats received post-surgical subcutaneous injections of $1.3 \mathrm{mg}, 3.9 \mathrm{mg}$ or $6.5 \mathrm{mg}$ buprenorphine per kg body weight (two times, six times or ten times the intended dose, respectively), and their body weight, clinical signs and symptoms, clinical pathology and histopathology were monitored for $4 \mathrm{~d}$. Body weight was not significantly different in rats that received buprenorphine compared with control rats. Signs of nausearelated behavior were observed in $25 \%$ of the rats treated with buprenorphine. Clinical pathology results for all rats were normal, and gross and microscopic histopathology examinations identified no substantial abnormalities, suggesting that this behavior was of minor consequence. Other adverse events previously reported to occur with opiate therapy, including weight loss and dermal lesions at drug injection sites, were not observed in this study. The results of this study show that post-surgical administration of an extended-release buprenorphine product is safe in Fischer 344 rats and does not necessarily cause substantial adverse effects, confirming that opiate therapy is a viable choice in laboratory animal medicine.

Pain management for laboratory rodents often requires repeated restraint of the animals and injection of analgesic drugs at intervals of 6-8 h, which can cause stress ${ }^{1}$ and potential injury. Analgesics that have longer durations of activity ${ }^{2}$ can allow investigators to manage pain in laboratory animals with a single dose of drug, minimizing potential stress and injury. This is a goal of long-acting opiate therapy. Previous research has established the efficacy of opiates such as buprenorphine in animals ${ }^{3-5}$. Buprenorphine has a high therapeutic index and a 35-year history of safe use in veterinary medicine ${ }^{6,7}$. Moreover, because opiates are a front-line therapy for pain management in humans, their use in laboratory animals comports with the objective of harmonizing pharmacology between laboratory models and human diseases ${ }^{8}$. Despite these advantages, opiate analgesia is rarely used with laboratory animals for various reasons ${ }^{9}$, including its potential to cause adverse effects. Reported adverse effects of opiate therapy include weight loss ${ }^{10-12}$, nausea ${ }^{13-16}$ and dermal lesions at drug injection sites ${ }^{17-22}$.

Extended-release buprenorphine has a defined safety profile in mice ${ }^{23}$, but a comprehensive understanding of its safety profile in rats is lacking. We aimed to determine whether an extended-release cholesterol-buprenorphine suspension could be used safely in rats. When designing safety studies for veterinary pharmaceutical products, the US Food and Drug Administration advises investigators to follow guidance for target animal safety (TAS) studies ${ }^{24,25}$. This guidance

${ }^{1}$ Temple University School of Medicine, Department of Pharmacology, Philadelphia, PA. ${ }^{2}$ Johns Hopkins School of Medicine, Department of Neurological Surgery, Baltimore, MD. ${ }^{3}$ WelCom Associates, Middletown, DE. ${ }^{4}$ George Mason University, Fairfax, VA. Correspondence should be addressed to M.G. (mguarnieri@comcast.net). 
indicates that TAS studies should include clinical observations for pain and distress as well as complete clinical pathology and histopathology examinations ${ }^{24,25}$. European regulatory authorities use similar guidelines to determine the effects of drugs on major organ systems in target animal species ${ }^{26}$. In TAS studies, animals are often challenged by administration of two- to ten-fold overdoses of the drug of interest in order to expose weak drug detoxification systems, potential risks in animal models of disease, and potential outcomes of accidental overdoses in the real world.

We designed and carried out a TAS study for postsurgical administration of extended-release buprenorphine in male and female Fischer 344 rats. The intended dose of extended-release buprenorphine for rodents is $0.65 \mathrm{mg}$ per $\mathrm{kg}$ body weight, as indicated on the FDA-approved product label. We evaluated the safety of three different doses of buprenorphine: $1.3 \mathrm{mg}$ per $\mathrm{kg}$ body weight (two-fold overdose), $3.9 \mathrm{mg}$ per $\mathrm{kg}$ body weight (six-fold overdose) or $6.5 \mathrm{mg}$ per $\mathrm{kg}$ body weight (ten-fold overdose). We administered buprenorphine or a control suspension to rats immediately after surgery and measured body weight, made clinical observations and analyzed clinical pathology over the next $4 \mathrm{~d}$. We also analyzed gross and microscopic histopathology at $4 \mathrm{~d}$ after surgery. Here we report some of these results with a particular focus on adverse events associated with these overdoses of extended-release buprenorphine in rats.

\section{METHODS}

\section{Animals and husbandry}

This study was approved by the IACUC of Johns Hopkins University. We obtained 32 Fischer 344 rats (16 male, 16 female) aged 6-8 weeks and weighing 180-220 g from Taconic Farms (Hudson, NY). Rats were housed in an environmentally controlled room with temperature of $20-26^{\circ} \mathrm{C}$ and relative humidity of $30-70 \%$ on a 12 -h:12-h light:dark cycle (with the lights coming on at 6:00 a.m.). Health surveillance was carried out using a soiled-bedding sentinel system, which indicated that rats were negative for pneumonia virus of mice, reovirus, Sendai virus, lymphocytic choriomeningitis virus, rat coronavirus, sialodacryoadenitis virus, rat parvovirus, Kilham rat virus, Toolan $\mathrm{H} 1$ parvovirus, rat theilovirus, cilia-associated respiratory bacillus, Pneumocysti carinii, Mycoplasma pulmonis and pinworms.

Upon their arrival at our facility, rats were quarantined and acclimated to the facility for $6 \mathrm{~d}$, during which no disease-related signs were noted. Rats were then evaluated and approved for study use by a clinical veterinarian. After the acclimation period we weighed the rats on a balance (Explorer Pro EP4102C, Ohaus, Parsippany, NJ) and randomized them by body weight using random numbers generated by Excel (Microsoft
Corporation, Redmond, WA) into four groups of eight rats each (four male, four female). The weight of each rat was within $10 \%$ of the mean weight of its group. At the start of the test the mean body weight of all females was approximately $130 \mathrm{~g}$. The mean body weight of all males was approximately $180 \mathrm{~g}$, with the exception of one heavier group that weighed a mean body weight of approximately $195 \mathrm{~g}$. Rats were tagged with unique ear tags (Kent Scientific, Torrington, CT) for identification and were housed in groups of two or three with cagemates of the same sex that were assigned to the same experimental group. Rats were housed in individually ventilated cages (Innovive, San Diego, CA) during the quarantine period and were housed individually in the same type of cage during the study period. Rats were provided with soft fiber bedding (Carefresh Natural, Ferndale, WA), ad libitum access to drinking water (from municipal sources) in disposable water bottles (Aquavive Rat $850 \mathrm{~mL}$, Innovive, San Diego, CA) and ad libitum access to Certified Global Rodent Diet 2016C (Harlan TEKLAD, Indianapolis, IN). Rats were also provided polycarbonate red tubes (Bio Services, Uden, The Netherlands) as enrichment devices.

\section{Study design}

We carried out sham surgeries for all the rats on day 0 and administered a single subcutaneous injection of buprenorphine or control suspension immediately after surgery. Rats in each of the four experimental groups ( $n=8$ per group, 4 male, 4 female) received a different injection: the control suspension, $1.3 \mathrm{mg}$ buprenorphine per kg body weight, $3.9 \mathrm{mg}$ buprenorphine per $\mathrm{kg}$ body weight or $6.5 \mathrm{mg}$ buprenorphine per $\mathrm{kg}$ body weight. The heavier group of males was included in the group that received the dose of $3.9 \mathrm{mg}$ per $\mathrm{kg}$ body weight. The study period lasted $4 \mathrm{~d}$ after the surgery and injection. We made clinical observations twice per day on days 1-3 and once on day 4, and we measured body weight and collected blood and urine samples on day 2 and day 4 . On day 4 , at the end of the study, we euthanized the rats.

\section{Surgical procedure}

We began surgery at approximately 7:00 a.m. and used a surgical procedure that mimics the subcutaneous implantation of a pump or recording device. We anesthetized each rat using an anesthesia machine (E-Z Anesthesia, Palmer, PA) with isoflurane gas at approximately $3 \%$ and oxygen at $1 \%$ (exact flow rate not recorded). We then shaved the scapular region (between the shoulder blades), washed it with ethanol and painted it with povidone-iodine solution. We transferred the rat to a clean procedural area and assessed the depth of anesthesia by pinching the toe. Failure to respond to the toe pinch indicated that the rat had reached a surgical plane of anesthesia. Once this had occurred, we visually 
assessed and documented the rat's respiratory rate and capillary fill rate after pinching a toenail. We used clean, sterilized forceps to gently grasp the skin, and clean, sterilized scissors to make a 4- to 5-mm incision at the scapular region through the skin without penetrating the muscle or bone. We then used the scissors to separate the skin and create a subcutaneous pocket measuring approximately $2 \mathrm{~cm} \times 4 \mathrm{~cm}$. We apposed the edges of the incision and stapled them closed using a 9-mm Autoclip (Kent Scientific, Torrington, CT). We documented the rat's respiratory rate and capillary refill time at the end of the procedure.

\section{Drug administration and post-surgical recovery}

Immediately after surgery and before the rat recovered from anesthesia, we administered the designated dose of control suspension or buprenorphine suspension to each rat. The control suspension consisted of cholesterol and glycerol tristearate (96:4) suspended in a mediumchain triglyceride oil ( $8 \mathrm{mg}$ per $100 \mu \mathrm{l}$; Animalgesics Labs, Millersville, MD). The buprenorphine suspension consisted of buprenorphine, cholesterol and glycerol tristearate suspended in a medium-chain triglyceride oil (Animalgesics for Mice; Animalgesics Labs, Millersville, MD). We administered the dose by subcutaneous injection in the mid-dorsal area about $1 \mathrm{~cm}$ rostral to the surgical incision using a 25 -gauge needle attached to a $1-\mathrm{ml}$ syringe (BD, Franklin Lakes, NJ). We then transferred the rat to a recovery cage and placed it on a heating pad to recover. Once the rat regained consciousness, began moving normally and showed no signs of distress, it was returned to its home cage.

\section{Clinical observations}

Clinical observations were carried out by two male observers certified by the American Association for Laboratory Animal Science, one as a Lab Animal Technologist and one as a Lab Animal Technician, who were blinded to experimental group. During each observation period one of these two observers would observe the rats and, afterward, complete the appropriate report form for either morning or afternoon observations, noting the presence of clinical signs or symptoms and adding comments as needed. Morning observations took place before 9:00 a.m. on days 1-4, and rats were observed without being removed from their cages. Observers noted morbidity; mortality; signs of pain or stress including abnormal respiration, tremors, ocular discharge, facial expression (squinting, eyes closed), posture and movement; overall appearance including condition of the hair coat; and condition of the incision site (bleeding, swelling and signs of infection). Afternoon observations took place after 2:00 p.m. on days $1-3$, and rats were removed from their cages and observed individually for 3-5 $\mathrm{min}$ each. Observers noted abnormal clinical signs (ocular discharge, motor activity and signs of pain or distress); the condition of the incision site (bleeding, edema, inflammation and signs of infection); and condition of the entire skin surface including hair loss and lesions.

Observers noted in comments on the report form any hair loss and the presence of lesions as evidence of excessive grooming or self-gnawing behavior, but they did not grade the amount of hair loss or the degree of biting. We considered any reports of hair loss or lesions on the paws to be signs of nausea-related behavior, and we recorded the number of rats of each sex in each experimental group that showed these signs during each observation period.

\section{Body weights}

An observer who was blinded to experimental group weighed each rat on a balance on days 0,2 and 4 at approximately 6:30 a.m. Weights are expressed as mean \pm s.d.

\section{Clinical pathology}

We collected samples of about $0.5 \mathrm{ml}$ blood from each rat on the mornings of days 2 and 4 via jugular vein puncture. We then refrigerated blood samples at $3-8{ }^{\circ} \mathrm{C}$ until they were delivered for analysis. We measured buprenorphine concentrations in blood samples from three rats of each sex that received $1.3 \mathrm{mg}$ buprenorphine per kg body weight, which satisfied the minimum sample size required by the US Food and Drug Administration for a pharmacokinetic study. We used a Shimadzu LC-20AD (Columbia, MD) and Applied Biosystems 4000 QTrap (Carlsbad, CA) to carry out a liquid chromatography-mass spectroscopy assay that requires $0.25 \mathrm{ml}$ of plasma ${ }^{23}$. We also carried out hematology tests on blood samples from all rats using a Hemavet 950 Hematology System (Drew Scientific, Waterbury, CT) and we carried out chemistry panels on blood samples from all rats using a VetACE Clinical Chemistry system (Alfa Wassermann, West Caldwell, NJ). We collected urine samples from each rat on the afternoons of day 2 and day 4 by placing the rat on a clean surface, expressing urine and transferring it to a sterile centrifuge tube using a pipette. We then analyzed the urine using urine dip sticks (Bayer Multistix 10 SG Reagent Strips, Romeoville, IL) and visually assessed the assays for abnormal parameters.

\section{Histopathology}

On the afternoon of day 4, we euthanized the rats by carbon dioxide inhalation in custom-made plastic chambers. We then carried out a thoracotomy and confirmed death by cessation of heart beat. We carried out a comprehensive necropsy for each rat as described previously $^{23,25}$, collecting 33 tissues and organs for histopathological examination, including the dorsal skin surrounding the injection site, for the complete 
TAS study. We weighed organs; incised the stomachs and examined the contents; and examined the entire gastrointestinal tract for any signs of blockage, distension, inflammation, edema or necrosis.

\section{Statistical analysis}

We carried out separate one-way analysis of variance tests (ANOVAs) to compare body weights between groups for rats within each sex for each day. We made pairwise comparisons between groups within sexes and within each day using Dunnett's post hoc tests. We used a zero-inflated Poisson regression model ${ }^{27}$ to estimate how sex and experimental group influenced the incidence of nausea-related behavior. We used zero-inflation to account for control rats that did not receive buprenorphine. We also used one-way ANOVAs and Dunnett's post hoc tests to compare organ weights and clinical pathology parameters between groups for rats of each sex as part of the TAS study, whose complete results are not described here. For all analyses $P<0.05$ was considered statistically significant. We carried out calculations and modeling using the $\mathrm{R}$ language version 3.0.2 (R Foundation for Statistical Computing, Vienna, Austria).

\section{RESULTS}

\section{Body weight}

Body weight was not significantly different between groups of either sex during the study $\left(F_{3,12}<3.4, P>0.05\right.$, Table 1). Male rats that received $1.3 \mathrm{mg}$ buprenorphine per kg body weight generally lost weight between day 0 and day 2, but generally gained weight between day 2 and day 4 . Female rats that received $1.3 \mathrm{mg}$ buprenorphine per kg body weight generally lost weight between day 0 and day 2 , and also lost weight between day 2 and day 4. All male rats that received $3.9 \mathrm{mg}$ buprenorphine per kg body weight lost weight between day 0 and day 2 . Two of these males continued to lose weight between day 2 and day 4 , whereas the other two males gained weight between day 2 and day 4 and had an overall weight gain from day 0 to day 4 . Among female rats that received $3.9 \mathrm{mg}$ buprenorphine per $\mathrm{kg}$ body weight, one lost weight from day 0 to day 2 and all four lost weight between day 2 and day 4, but only two had an overall weight loss from day 0 to day 4 . All male rats that received $6.5 \mathrm{mg}$ buprenorphine per kg body weight lost weight between day 0 and day 2 but gained weight between day 2 and day 4 . On the other hand, all of the female rats treated with $6.5 \mathrm{mg}$ buprenorphine per $\mathrm{kg}$ body weight gained weight between day 0 and day 2 but lost weight between day 2 and day 4 .

\section{Clinical observations}

Observers noted no signs of pain or distress in any of the animals as part of the TAS study.

Signs of nausea-related behavior were never observed during the morning observation periods but were observed during the afternoon periods when each rat was removed from its cage for physical examination. These signs were observed only on the forepaws. No signs of nausea-related behavior were observed in control rats. Signs of nausea-related behavior were first noted in a male rat $1 \mathrm{~d}$ after receiving an injection of $3.9 \mathrm{mg}$ buprenorphine per $\mathrm{kg}$ body weight (Table 2). Among rats that received the lowest dose of $1.3 \mathrm{mg}$ buprenorphine per $\mathrm{kg}$ body weight, only one female rat showed signs of nausea-related behavior on day 2 and on day 3. Among rats that received $3.9 \mathrm{mg}$ buprenorphine per kg body weight, the incidence of signs of nausea-related behavior increased during the study from one male on day 1 to two rats (one male and one female) on day 2 and three rats (one male and two females) on day 3. The incidence of signs of nausearelated behavior was highest in this experimental group. Among rats that received the highest dose of $6.5 \mathrm{mg}$ buprenorphine per kg body weight, the onset of signs of nausea-related behavior was delayed and the incidence was lower: two rats (one male, one female) showed signs of nausea-related behavior on day 3. Across the three groups of rats that received buprenorphine, 6 of 24 rats (25\%) showed signs of nausea-related behavior on day 3. Treatment group was a statistically significant predictor of the number of rats that showed signs of nausearelated behaviors $(\beta=1.01$, standard error (s.e.) $=0.41$, $P=0.01)$. The incidence of signs of nausearelated behavior was similar in male and female rats

TABLE 1 | Mean \pm s.d. body weight (g) of male and female rats at day 0, 2 and 4. Single-sex groups of rats $(n=4)$ received either a control injection or one of three different doses of buprenorphine. Body weight did not differ significantly between groups of male or female rats at any point during the study.

\begin{tabular}{|c|c|c|c|c|c|c|}
\hline \multirow{2}{*}{$\begin{array}{l}\text { Buprenorphine } \\
\text { dose (mg per kg } \\
\text { body weight) }\end{array}$} & \multicolumn{3}{|c|}{ Male } & \multicolumn{3}{|c|}{ Female } \\
\hline & Day 0 & Day 2 & Day 4 & Day 0 & Day 2 & Day 4 \\
\hline 0 & $170.8 \pm 15.9$ & $172.3 \pm 14.4$ & $177.3 \pm 13.6$ & $130.0 \pm 8.1$ & $129.8 \pm 7.4$ & $132.1 \pm 9.8$ \\
\hline 1.3 & $177.2 \pm 9.0$ & $175.1 \pm 8.5$ & $178.7 \pm 7.3$ & $129.5 \pm 12.9$ & $128.7 \pm 12.9$ & $127.5 \pm 12.4$ \\
\hline 3.9 & $195.9 \pm 21.4$ & $192.9 \pm 19.2$ & $192.7 \pm 17.9$ & $132.9 \pm 8.6$ & $134.1 \pm 10.5$ & $133.2 \pm 10.6$ \\
\hline 6.5 & $170.8 \pm 15.9$ & $172.3 \pm 14.4$ & $177.3 \pm 13.6$ & $130.0 \pm 8.1$ & $129.8 \pm 7.4$ & $132.1 \pm 9.8$ \\
\hline
\end{tabular}




\begin{tabular}{|c|c|c|c|c|c|}
\hline $\begin{array}{l}\text { Buprenorphine dose } \\
\text { (mg per kg body weight) }\end{array}$ & $n$ & Day 0 & Day 1 & Day 2 & Day 3 \\
\hline 0 & 8 ( 4 male, 4 female) & 0 & 0 & 0 & 0 \\
\hline 1.3 & 8 ( 4 male, 4 female) & 0 & 0 & 1 female & 1 female \\
\hline 3.9 & 8 ( 4 male, 4 female) & 0 & 1 male & 2 (1 male, 1 female) & 3 (1 male, 2 female) \\
\hline 6.5 & 8 (4 male, 4 female) & 0 & 0 & 0 & 2 (1 male, 1 female) \\
\hline
\end{tabular}

and sex was not a statistically significant predictor of the number of rats that showed signs of nausea-related behavior $(\beta=-0.406$, s.e. $=0.65, P=0.53)$.

\section{Clinical pathology}

Blood concentrations of buprenorphine in rats that received $1.3 \mathrm{mg}$ buprenorphine per $\mathrm{kg}$ body weight averaged $5,7,5$ and $4 \mathrm{ng} / \mathrm{ml}$ on days $1-4$, respectively, for males and averaged 10, 7, 7 and $2 \mathrm{ng} / \mathrm{ml}$ on days 1-4, respectively, for females. Clinical pathology data showed no significant differences between the control and drug treated groups. Most animals in all groups, including the negative controls, had moderate levels of protein in the urine that did not discernibly differ between doses. Hematology and clinical chemistry analyses identified no abnormal blood chemistry that could indicate renal damage.

\section{Pathology}

Necropsy examinations identified no gross abnormalities, including macroscopic skin lesions, in any of the rats. Among all organs, only spleen weight differed significantly among groups $\left(F_{3,12}=3.96, P=0.036\right)$, but this was not considered indicative of an adverse effect. Microscopic examination identified no abnormalities in dorsal skin tissue, spleen or any other tissues or organs from rats in any experimental group. Post-mortem examination of the stomach contents and gastrointestinal tracts showed no anomalies or signs of blockage, distension, inflammation, edema or necrosis in any of the rats.

\section{DISCUSSION}

The efficacy of long-acting opiate analgesics in laboratory animals has been established ${ }^{2}$, but less information is available about the safety of these veterinary pharmaceuticals. In this study, we examined whether an extended-release buprenorphine suspension with a defined safety profile in mice ${ }^{23}$ could be used safely in rats. We administered three different doses of buprenorphine to rats (1.3 mg per kg body weight, $3.9 \mathrm{mg}$ per $\mathrm{kg}$ body weight or $6.5 \mathrm{mg}$ per $\mathrm{kg}$ body weight) immediately after surgery and measured body weight, made clinical observations and analyzed clinical pathology and histopathology over the next $4 \mathrm{~d}$ to identify adverse events including weight loss ${ }^{10-12}$, nausea ${ }^{13-16}$ and dermal lesions at drug injection sites ${ }^{17-22}$, which have previously been reported to occur with opiate therapy.

Blood concentrations of buprenorphine in rats that received the lowest dose of $1.3 \mathrm{mg}$ buprenorphine per $\mathrm{kg}$ body weight exceeded the threshold of $1 \mathrm{ng} / \mathrm{ml}$ that has been consistently associated with analgesic effi$\mathrm{cacy}^{2,7}$. Body weight was not significantly different in rats that received buprenorphine compared with control rats of either sex during the study. Signs of nausea-related behavior were observed in $25 \%$ of the rats treated with buprenorphine. Clinical pathology results for all rats were normal, and gross and microscopic histopathology examinations identified no substantial abnormalities.

We did not observe significant weight loss in rats that received buprenorphine in this study. A similar absence of weight loss was also reported in rats that received $1.2 \mathrm{mg}$ buprenorphine per $\mathrm{kg}$ body weight in a polymer formulation ${ }^{19,28}$. These results contrast with previous reports of significant weight loss after post-surgical oral and subcutaneous administration of buprenorphine ${ }^{10-12}$. Weight loss in some cases was associated with gastric distress and pica (consumption of non-nutritive substances) ${ }^{10,11}$. Pica occurs in response to nausea induced by opiate therapy ${ }^{3,10}$ and has been reported in Sprague-Dawley ${ }^{13,16}$, Long-Evans ${ }^{15}$, Wistar $^{14}$ and Dark Agouti rats ${ }^{12}$. Pica is a complex phenomenon that might be beneficial in some circumstances. Consumption of kaolin (a behavior indicative of pica) after treatment with a nausea-inducing compound was correlated with less weight loss and greater food consumption in rats ${ }^{29}$. Pica can also have dangerous consequences, however. Previously in our laboratory, 1 of 15 male Fischer 344 rats was found dead on day 3 of a pilot study after it was treated with a single dose of $1.3 \mathrm{mg}$ buprenorphine per $\mathrm{kg}$ body weight. Upon necropsy, the stomach and esophagus of the rat were noted to be compacted with hardwood bedding. This is consistent with a previous report that Sprague-Dawley rats consumed hardwood bedding and experienced gastric distention after treatment with buprenorphine ${ }^{16}$. In another study in which rats were housed on corncob bedding, pica-related mortality was reported in 5 of 25 animals treated post-surgically with buprenorphine or fentanyl, but no deaths occurred among 30 control rats 
or 20 rats treated with acetaminophen or flunixin ${ }^{30}$. Another study reported no deaths in rats treated with buprenorphine and housed for 1-2 d on grid floors or on paper ${ }^{12}$. In this study, we observed no deaths and no signs of pica among 32 rats treated with buprenorphine and housed with soft fiber bedding. These results suggest that, although opiate-induced pica can have severe and even lethal consequences for rats housed with hardwood and corncob bedding ${ }^{13,14}$, these can be minimized if researchers instead use alternative bedding materials, such as soft fiber.

Although we did not note weight loss or signs of pica in this study, we did observe signs of nausea-related behavior in rats treated with buprenorphine. Nausea is a common adverse effect of opiate therapy in humans and animals and can confound experimental results. We observed signs of nausea-related behavior in $25 \%$ of the rats treated with buprenorphine. This incidence is similar to the incidence of nausea reported among human patients treated with opiate therapies ${ }^{31}$. The male rat that first showed signs of nausea-related behavior on day 1 was one of two male rats receiving $3.9 \mathrm{mg}$ buprenorphine per $\mathrm{kg}$ body weight that lost weight throughout the study. Clinical pathology results for all rats were normal, however, suggesting that consequences of this behavior were minor. The presence of moderate levels of protein in expressed urine could generally indicate renal damage, but this was not supported by the blood chemistry findings.

Signs of nausea-related behavior were not observed in a similar TAS study of the safety of buprenorphine in mice $^{23}$, and we are unaware of previous reports describing excessive grooming and self-gnawing behavior associated with long-acting buprenorphine therapy in laboratory animals. Whether the signs we observed (hair loss and lesions on the forepaws) in this study are entirely related to nausea requires further verification. Future studies of nausea-related behavior in rats treated with buprenorphine are also needed to address whether its incidence can be reduced by lower doses of drug; to assess its incidence, duration and intensity; and to determine whether it occurs in other rat strains ${ }^{32,33}$. The use of light and dark surveillance ${ }^{9}$ and automated tracking ${ }^{34}$ techniques in such studies might help to better characterize this behavior.

Dermal lesions have been reported at the sites of subcutaneous injections of buprenorphine-loaded polymers in mice ${ }^{17,18}$, $\operatorname{rats}^{19}, \operatorname{dogs}^{20}$, minipigs ${ }^{21}$ and nonhuman primates ${ }^{22}$ but were not observed at the site of subcutaneous injection of a cholesterolbuprenorphine suspension in mice ${ }^{23}$. Lipid encapsulation is a well-known mechanism used to decrease toxicity and prolong delivery of drugs ${ }^{35}$. In this study we observed no skin lesions upon gross examination and histopathologic analysis of the site of subcutaneous injection of the same cholesterol-buprenorphine suspension in rats.

To our knowledge, the previous report on the safety of an extended-release buprenorphine suspension describes the first use of a TAS study to evaluate a drug specifically designed for mice ${ }^{23}$. This study used a similar protocol to examine the safety of the drug in rats. The results indicate that post-surgical administration of extended-release buprenorphine is safe and does not cause substantial adverse effects in relatively young Fischer 344 rats of both sexes under appropriate husbandry conditions. The safety of the drug in older rats, in rats of different strains and in the context of different surgical procedures is unknown. Yet we conclude that long-acting opiate therapy is a viable analgesic strategy in laboratory animal medicine and, with appropriate husbandry, could markedly expand options for providing post-surgical pain therapy to laboratory rats.

\section{ACKNOWLEDGMENTS}

We thank James Kehler, VMD, PhD for his advice in preparing this manuscript. Funding for this research was supplied by The Maryland Biotechnology Center Biotechnology Development Awards and by Animalgesics Labs.

\section{COMPETING INTERESTS STATEMENT}

The authors declare competing financial interests. M.G. owns a significant financial interest in Animalgesics Labs.

Received 21 March 2014; accepted 24 February 2015 Published online at http://www.labanimal.com/

1. Schaap, M.W. et al. Optimizing the dosing interval of buprenorphine in a multimodal postoperative analgesic strategy in the rat: minimizing side-effects without affecting weight gain and food intake. Lab. Anim. 46, 287-292 (2012).

2. Foley, P.L. Current options for providing sustained analgesia to laboratory animals. Lab Anim. (NY) 43, 364-371 (2014).

3. Cowan, A., Doxey, J.C. \& Harry, E.J. The animal pharmacology of buprenorphine, an oripavine analgesic agent. Br. J. Pharmacol. 60, 547-554 (1977).

4. Cowan, A., Lewis, J.W. \& MacFarlane, I.R. Agonist and antagonist properties of buprenorphine, a new antinociceptive agent. Br. J. Pharmacol. 60, 537-545 (1977).

5. Yassen, A., Olofsen, E., Kan, J., Dahan, A. \& Danhof, M. Pharmacokinetic-pharmacodynamic modeling of the effectiveness and safety of buprenorphine and fentanyl in rats. Pharm. Res. 25, 183-193 (2008).

6. Roughan, J.V. \& Flecknell, P.A. Buprenorphine: a reappraisal of its antinociceptive effects and therapeutic use in alleviating post-procedural pain in animals. Lab. Anim. 36, 322-343 (2002).

7. Guarnieri, M. et al. Safety and efficacy of buprenorphine for analgesia in laboratory mice and rats. Lab Anim. (NY) 41, 337-343 (2012).

8. Percie du Sert, N. \& Rice, A.S. Improving the translation of analgesic drugs to the clinic: animal models of neuropathic pain. Br. J. Pharmacol. 171, 2951-2963 (2014).

9. Adamson, T.W. et al. Assessment of carprofen and buprenorphine on recovery of mice after surgical removal of the mammary fat pad. J. Am. Assoc. Lab. Anim. Sci. 49, 610-616 (2010). 
10. Aung, H.H., Mehendale, S.R., Xie, J.T., Moss, J. \& Yuan, C.S. Methylnaltrexone prevents morphine-induced kaolin intake in the rat. Life Sci. 74, 2685-2691 (2004).

11. Batra, V.R. \& Schrott, L.M. Acute oxycodone induces the pro-emetic pica response in rats. J. Pharmacol. Exp. Ther. 339, 738-745 (2011).

12. Jablonski, P., Howden, B.0. \& Baxter, K. Influence of buprenorphine analgesia on post-operative recovery in two strains of rats. Lab. Anim. 35, 213-222 (2001).

13. Jacobson, C. Adverse effects on growth rates in rats caused by buprenorphine administration. Lab. Anim. 34, 202-206 (2000).

14. Bender, H.M. Letter to the Editor. Lab. Anim. Sci. 48, 5 (1998).

15. Thompson, A.C. et al. Analgesic efficacy of orally administered buprenorphine in rats: methodologic considerations. Comp. Med. 54, 293-300 (2004).

16. Clark, J.A. Jr., Myers, P.H., Goelz, M.F., Thigpen, J.E. \& Forsythe, D.B. Pica behavior associated with buprenorphine administration in the rat. Lab. Anim. Sci. 47, 300-303 (1997).

17. Clark, T.S., Clark, D.D. \& Hoyt, R.F. Jr. Pharmacokinetic comparison of sustained-release and standard buprenorphine in mice. J. Am. Assoc. Lab. Anim. Sci. 53, 387-391 (2014).

18. Carbone, E.T., Lindstrom, K.E., Diep, S. \& Carbone, L. Duration of action of sustained-release buprenorphine in 2 strains of mice. J. Am. Assoc. Lab. Anim. Sci. 51, 815-819 (2012).

19. Foley, P.L., Liang, H. \& Crichlow, A.R. Evaluation of a sustained release formulation of buprenorphine for analgesia in rats. J. Am. Assoc. Lab. Anim. Sci. 50, 198-204 (2011).

20. Nunamaker, E.A. et al. Clinical efficacy of sustained-release buprenorphine with meloxicam for postoperative analgesia in beagle dogs undergoing ovariohysterectomy. J. Am. Assoc. Lab. Anim. Sci. 53, 494-501 (2014).

21. Thiede, A.J. et al. Pharmacokinetics of sustained-release and transdermal buprenorphine in Göttingen minipigs (Sus scrofa domestica). J. Am. Assoc. Lab. Anim. Sci. 53, 692-699 (2014).

22. Nunamaker, E.A. et al. Pharmacokinetics of 2 formulations of buprenorphine in macaques (Macaca mulatta and Macaca fascicularis). J. Am. Assoc. Lab. Anim. Sci. 52, 48-56 (2013).

23. Traul, K.A. et al. Safety studies of post-surgical buprenorphine therapy for mice. Lab. Anim. 49, 100-110 (2015).
24. US Food and Drug Administration. Guidance for Industry: Target Animal Safety for Veterinary Pharmaceutical Products. (US Food and Drug Administration, Silver Spring, MD, 2009). http://www.fda.gov/downloads/AnimalVeterinary/ GuidanceComplianceEnforcement/GuidanceforIndustry/ ucm052464.pdf.

25. US Food and Drug Administration. Guidance for Industry: FDA Approval of New Animal Drugs for Minor Uses and for Minor Species. (US Food and Drug Administration, Silver Spring, MD, 2008). http://www.fda.gov/downloads/AnimalVeterinary/ GuidanceComplianceEnforcement/GuidanceforIndustry/ ucm052375.pdf.

26. Guidelines of Target Animal Safety for Pharmaceuticals, VICH Topic GL43, European Medicines Agency Veterinary Medicines and Inspections. November (2006)

27. Lambert, D. Zero-inflated Poisson regression, with an application to defects in manufacturing. Technometrics 34, 1-14 (1992).

28. Chum, H.H. et al. Antinociceptive effects of sustainedrelease buprenorphine in a model of incisional pain in rats (Rattus norvegicus). J. Am. Assoc. Lab. Anim. Sci. 53, 193-197 (2014).

29. De Jonghe, B.C., Lawler, M.P., Horn, C.C. \& Tordoff, M.G. Pica as an adaptive response: Kaolin consumption helps rats recover from chemotherapy-induced illness. Physiol. Behav. 97, 87-90 (2009).

30. Stewart, L.S.A. \& Martin, W.J. Evaluation of postoperative analgesia in a rat model of incisional pain. Contemp. Top. Lab. Anim. Sci. 42, 28-34 (2003).

31. Aparasu, R., McCoy, R.A., Weber, C., Mair, D. \& Parasuraman, T.V. Opioid-induced emesis among hospitalized nonsurgical patients: effect on pain and quality of life. J. Pain Symptom Manage. 18, 280-288 (1999).

32. Morgan, D., Cook, C.D. \& Picker, M.J. Sensitivity to the discriminative stimulus and antinociceptive effects of mu opioids: role of strain of rat, stimulus intensity, and intrinsic efficacy at the mu opioid receptor. J. Pharmacol. Exp. Ther. 289, 965-975 (1999).

33. Mitchell, D., Krusemark, M.L. \& Hafner, D. Pica: a species relevant behavioral assay of motion sickness in the rat. Physiol. Behav. 18, 125-130 (1977).

34. Catarinucci, L. et al. An animal tracking system for behavio analysis using radio frequency identification. Lab Anim. (NY) 43, 321-327 (2014).

35. Mishra, D.K., Dhote, V., Bhatnagar, P. \& Mishra, P.K. Engineering solid lipid nanoparticles for improved drug delivery: promises and challenges of translational research. Drug Deliv. Transl. Res. 2, 238-253 (2012). 